\title{
CARACTERIZAÇÃO DAS ESPÉCIES FLORESTAIS EM PLANOS DE MANEJO FLORESTAL SUSTENTÁVEL EM PEQUENA ESCALA NO ESTADO DO AMAZONAS
}

\author{
CHARACTERIZATION OF FOREST SPECIES IN SMALL-SCALE SUSTAINABLE FOREST \\ MANAGEMENT PLANS IN THE STATE OF AMAZONAS
}

\author{
Filipe Campos Freitas ${ }^{1}$, Eirie Gentil Vinhote ${ }^{2}$, Alberto Carlos Martins Pinto ${ }^{3}$ \\ ${ }^{1}$ Instituto de Desenvolvimento Agropecuário e Florestal Sustentável do Estado do Amazonas, Manaus, \\ Amazonas, Brasil - filipe.freitas19@gmail.com \\ ${ }^{2}$ Secretaria de Estado do Meio Ambiente, Manaus, Amazonas, Brasil - evinhote@gmail.com \\ ${ }^{3}$ Universidade Federal do Amazonas, Manaus, Amazonas, Brasil - acmppinto@gmail.com
}

RESUMO

\begin{abstract}
O manejo em pequena escala é uma alternativa de uso dos recursos madeireiros para pequenos produtores no estado do Amazonas. Estudos florísticos são importantes para o conhecimento da flora regional e seus potenciais diversos. O objetivo deste estudo foi avaliar a composição florística e estrutura florestal em planos de manejo em pequena escala a fim de se conhecer quais espécies tem sido mais visada pelos pequenos produtores rurais do Amazonas e verificar se a diversidade de espécies dessas áreas condiz com o esperado para a região Amazônica. Foram utilizadas planilhas de inventário florestal de planos de manejo licenciados no ano de 2013. Foi realizada a análise da estrutura horizontal considerando os parâmetros de densidade, dominância, frequência e valor de importância das espécies. A diversidade da vegetação foi avaliada a partir dos índices de diversidade de Shannon-Wiener $\left(\mathrm{H}^{\prime}\right)$ e equabilidade de Pielou (J). Houve um total de 5716 indivíduos mensurados, representando 158 espécies e 35 famílias botânicas. As 10 famílias com maior riqueza de espécies foram Fabaceae (37), Lauraceae (18), Lecythidaceae (15), Sapotaceae (9), Moraceae (9), Chrysobalanaceae (8), Malvaceae (7) Myristicaceae (6), Anacardiaceae (5) e Caryocaraceae (4). As 10 espécies com maior valor de importância foram Micropholis williamii, Goupia glabra, Couratari tauari, Chrysophyllum L., Scleronema micranthum, Licania heteromorfa, Couepia subcordata, Tachigali paniculata, Peltogyne densiflora e Dipteryx odorata. A diversidade de espécies em planos de manejo em pequena escala condiz com o esperado para a ragião amazônica, sendo considerada alta.
\end{abstract}

PALAVRAS-CHAVE: Composição florística, Manejo comunitário, Produção madeireira.

\section{ABSTRACT}

Small-scale management is an alternative for the use of timber resources for small producers in the state of Amazonas. Floristic studies are important for the knowledge of the regional flora and its diverse potentials. The aim of this study was to evaluate the floristic composition and forest structure in small-scale management plans to know which species have been most targeted by small rural producers in Amazonas and to verify whether the diversity of species in these areas is consistent with the expected for the Amazon region. It was used forest inventory spreadsheets of management plans licensed in 2013. The analysis of the horizontal structure was carried out considering the parameters of density, dominance, frequency, and importance value of the species. Vegetation diversity was assessed using Shannon-Wiener $\left(\mathrm{H}^{\prime}\right)$ and Pielou $(\mathrm{J})$ indexes. There was a total of 5716 individuals measured, representing 158 species and 35 botanical families. The 10 families with the highest species richness were Fabaceae (37), Lauraceae (18), Lecythidaceae (15), Sapotaceae (9), Moraceae (9), Chrysobalanaceae (8), Malvaceae (7) Myristicaceae (6), Anacardiaceae (5) and Caryocaraceae (4). The 10 most important species were Micropholis williamii, Goupia glabra, Couratari tauari, Chrysophyllum L., Scleronema micranthum, Licania heteromorfa, Couepia subcordata, Tachigali paniculata, Peltogyne densiflora and Dipteryx odorata. The diversity of species in small-scale management plans is in line with what is expected for the Amazonian region, being considered high.

KEYWORDS: Community management, Floristic composition, Timber production. 


\section{INTRODUÇÃO}

O Capítulo VII da Lei 12651 de 2012 (Código Florestal Brasileiro) estabelece que a exploração de florestas nativas, destacando aqui aquelas localizadas na região amazônica, dependerá de elaboração e aprovação de Plano de Manejo Florestal Sustentável - PMFS (BRASIL, 2012). Apesar de uma lei relativamente recente, desde 1965 é estabelecido que as florestas primárias da Amazônia só poderiam ser alvo de exploração através de planos técnicos de manejo (BRASIL, 1965).

Em 1998, o Decreto 2788/98 regulamentou o uso de recursos florestais na Amazônia e possibilitou o licenciamento de forma comunitária em áreas de até 500 ha por meio de Plano de Manejo Florestal Sustentável Simplificado. Neste contexto, no ano de 2003, a Secretaria de Estado do Meio Ambiente e Desenvolvimento Sustentável do Amazonas elaborou a Portaria no 40/03 que dispõe sobre Planos de Manejo Florestal Sustentável em Pequena Escala (PMFSPE) com o objetivo de criar condições legais do manejo florestal para populações tradicionais (KLIBER, 2008). Atualmente, essa categoria é regulamentada pela Resolução no 007/2011 do Conselho Estadual de Meio Ambiente do Estado do Amazonas CEMAAM (AMAZONAS, 2011).

Desde a sua criação, essa categoria de manejo em pequena escala vem recebendo um número cada vez maior de interessados, a maioria sendo pequenos produtores rurais que se beneficiam com uma fonte a mais de renda. Como principal fator responsável pelo crescimento dessa categoria de manejo pode-se citar a assistência técnica gratuita oferecida pelo governo do estado, que auxilia o pequeno produtor nas fases técnicas e administrativas para elaboração e licenciamento do plano de manejo (FREITAS, 2014). Diante dessa realidade de crescimento pela procura por meios legais que possibilitem a utilização dos recursos madeireiros, se faz importante 0 conhecimento das espécies arbóreas de interesse comercial local que serão objeto de licenciamento para posterior exploração.

Com o seu território $100 \%$ inserido no bioma amazônico, o estado do Amazonas apresenta todas as características referentes a este ecossistema. A floresta amazônica é caracterizada como umas das poucas áreas que ainda detém os maiores níveis de biodiversidade do mundo, sendo constituída por diferentes tipos de vegetação, e cerca de $65 \%$ de sua área é coberta por um tipo florestal denominado floresta de terra firme (OLIVEIRA \& AMARAL, 2004). Assim, estudos florísticos e fitossociológicos da vegetação são importantes para o conhecimento da flora regional e seus potenciais diversos. Conhecimentos acerca desses assuntos são essenciais para a conservação da diversidade de uma floresta, e ainda permitem o planejamento e o estabelecimento de sistemas de manejo com produção sustentável (LIMA et al., 2012).

Desta forma, o presente estudo teve como objetivo avaliar a composição florística e estrutura florestal em PMFSPE a fim de se conhecer quais espécies tem sido mais visada pelos pequenos produtores rurais do Amazonas para obtenção de madeira e verificar se a diversidade das espécies alvo do manejo condiz com o esperado para a região Amazônica.

\section{MATERIAL E MÉTODOS}

\section{Área de estudo}

O estudo considerou as áreas com planos de manejo florestal em pequena escala licenciados para exploração no estado do Amazonas. Todas as áreas são constituídas por Floresta Ombrófila Densa em Terra Firme com indivíduos arbóreos de grande porte (IBGE, 2012). A classificação climática de Köppen para a região de abrangência do estudo é do tipo "Am" (tropical chuvoso monçônico). A precipitação média anual varia entre 1.355 e $2.839 \mathrm{~mm}$. Os meses mais chuvosos vão de dezembro a maio e os mais secos de agosto a novembro - precipitação mensal nunca inferior a $50 \mathrm{~mm}$. A temperatura média varia de $25,6^{\circ} \mathrm{C}$ a $27,6{ }^{\circ} \mathrm{C}$, com umidade relativa do ar média entre $84 \%$ e $90 \%$.

\section{Base de dados}

Os dados foram disponibilizados pelo Instituto de Desenvolvimento Agropecuário e Florestal Sustentável do Estado do Amazonas (IDAM), órgão público que oferece serviço de Assistência Técnica e Extensão Rural (ATER) aos pequenos produtores rurais do Estado. Foram utilizadas as planilhas de inventário florestal de planos de manejo licenciados no ano de 2013. Foi considerado esse ano por ter sido o com maior quantidade de licenças expedidas para exploração florestal dessa categoria de manejo.

Os inventários realizados para elaboração de PMFSPE, consistem num censo de uma pequena área da propriedade alvo do manejo, na proporção aproximada de $1 / 25$ da área total, com o intuito de assegurar o ciclo de corte de 25 anos estabelecido em legislação (AMAZONAS, 2011). Dessa forma, as áreas de inventário variam de acordo com o tamanho da propriedade. Para minimizar os 
efeitos dessa variação, foram selecionados para análise os inventários florestais realizados em áreas entre 10 e 12 ha. Dessa forma, foram considerados 27 inventários florestais, totalizando 299,27 hectares (média de 11,08 \pm 0,28 ha).

Previamente a ida à campo, foi realizada uma consulta prévia aos interessados no manejo em pequena escala (moradores da comunidade ou donos de propriedades) acerca das espécies mais ocorrentes e quais oferecem maior valor para comercialização. A identificação botânica das árvores foi feita por mateiros da região a partir de nome comum, os quais, posteriormente, foram cruzados com banco de dados do Software Árvore, do Ibama. A atualização dos nomes científicos das espécies listadas foi feita a partir de consulta ao Sistema de Informação sobre a Biodiversidade Brasileira (SiBBr).

Devido à legislação florestal, o principal interesse está nas árvores com diâmetro à altura do peito (DAP) a partir de $50 \mathrm{~cm}$, as quais podem ser selecionadas para corte (AMAZONAS, 2011). Dessa forma, não houve padronização quanto ao DAP mínimo para coleta de dados, sendo que em três inventários florestais o diâmetro mínimo esteve entre $20 \mathrm{~cm}$ e $29 \mathrm{~cm}$; em 12 o DAP mínimo esteve entre 30 $\mathrm{cm}$ e $39 \mathrm{~cm}$; e em outros 12 entre $40 \mathrm{~cm}$ e $49 \mathrm{~cm}$. Na Tabela 1 é apresentada a estatística descritiva da variação diamétrica observada nos inventários considerados nesse estudo.

Tabela 1. Estatística descritiva do Diâmetro à Altura do Peito em inventários florestais para elaboração de Planos de Manejo Florestal em Pequena Escala.

\begin{tabular}{cc}
\hline Variável & DAP \\
\hline Mínimo & $20 \mathrm{~cm}$ \\
Máximo & $270 \mathrm{~cm}$ \\
Médio & $58,7 \mathrm{~cm}$ \\
Desvio padrão & $14,7 \mathrm{~cm}$ \\
Coeficiente de variação & $25,0 \%$ \\
\hline
\end{tabular}

\section{Análise de dados}

Foi realizada a análise da estrutura horizontal considerando os parâmetros de densidade, dominância, frequência e valor de importância das espécies (MUELLERDOMBOIS; ELLENBERG, 1974). A diversidade da vegetação foi avaliada a partir dos índices de diversidade de ShannonWiener ( $H^{\prime}$ ) e equabilidade de Pielou (J) (SHANNON, 1948; PIELOU, 1966). Também foi realizada a categorização dos indivíduos em classes de diâmetros.

\section{RESULTADOS E DISCUSSÃO}

Foram inventariados um total de 5.716 indivíduos (15 \pm 2 ha $^{-1}$ ), representando 158 espécies e 35 famílias botânicas. As 10 famílias com maior riqueza de espécies foram Fabaceae (37), Lauraceae (18), Lecythidaceae (15), Sapotaceae (9), Moraceae (9), Chrysobalanaceae (8), Malvaceae (7) Myristicaceae (6), Anacardiaceae (5) e Caryocaraceae (4). Essas famílias representam $74,7 \%$ das espécies listadas. As demais famílias obtiveram menos de 4 espécies, sendo que 13 apresentaram penas uma espécie. Essa relação de famílias botânicas é semelhante a outros levantamentos realizados na região amazônica (ANDRADE et al., 2015; JARDIM \& QUADROS, 2016; BRANDÃO et al., 2020; VINHOTE et al., 2020).

As 10 espécies com maior valor de importância foram Micropholis williamii (Abiurana), Goupia glabra (Cupiúba), Couratari tauari (Tauari), Chrysophyllum L. (Abiurana), Scleronema micranthum (Cedrinho), Licania heteromorfa var. glabra (Macucu), Couepia subcordata (Marirana), Tachigali paniculata (Taxi), Peltogyne densiflora (Roxinho) e Dipteryx odorata (Cumaru) (Tabela 2). Essas espécies diferem das verificadas com maior valor de importância listada por outros pesquisadores (ANDRADE et al., 2015; BRANDÃO et al., 2020; JARDIM \& QUADROS, 2016; LIMA et al., 2018; VIEIRA et al., 2014). De forma geral, esses estudos citados mostram um predomínio de espécies do gênero Eschweilera entre as de maior importância. De acordo com Steege et al. (2013), de fato há um predomínio desse gênero na região Amazônica. Na Tabela 2, a primeira espécie desse gênero é verificada apenas na vigésima terceira posição. Essa diferença de espécies pode ser explicada pelos critérios de inclusão nos inventários florestais realizados, uma vez que no presente estudo foram incluídos apenas indivíduos de interesse por parte dos detentores de PMFSPE e sem padronização no diâmetro mínimo de coleta de dados (Figura 1).

Ao se analisar a densidade das espécies, verifica-se que, para as 10 principais listadas anteriormente, apenas Dipteryx odorata sai desse ranking, dando lugar a Pouteria guianensis, com 157 indivíduos. Para a dominância, o maior valor é apresentado pela Couratari tauari $(5,09)$, indicando que a espécie possui os maiores diâmetros; para esta variável as mesmas 10 espécies com maior VI são as de maior dominância.

Quanto à frequência, a espécie Goupia glabra foi a única a ser identificada em todos os inventários considerados desse estudo, ficando em primeiro lugar para essa variável. Por outro lado, a Micropholis williamii aparece em apenas $48 \%$ dos inventários, indicando que o 
alto valor de importância dessa espécie é devido, principalmente, à sua densidade e dominância. Nas 10 espécies de maiores frequências, há quatro espécies diferentes daquelas listadas quanto ao VI: Clarisia racemosa, Lecythis pisonis, Simarouba amara e Licaria guianensis.

Entre as 10 espécies de maior VI, verifica-se que duas são conhecidas pelo mesmo nome comum, Abiurana (Micropholis williamii e Chrysophyllum L.). Esse mesmo nome comum é atribuído a outros dois nomes científicos: Pouteria guianensis e Chrysophyllum prieurii. A espécie com terceiro maior VI, Tauari (Couratari tauari), também é associada a mais dois nomes científicos diferentes:
Cariniana micrantha e Couratari Aubl. Situações como essa são bastante comuns em PMFSPE, uma vez que a identificação é feita por mateiros de cada região a partir de características dendrológicas como: casca, copa, folha e posteriormente buscado um nome científico de acordo com área de ocorrência. Outros exemplos que podem ser destacados, em que mais de um nome científico é associado a um mesmo nome comum, sendo eles: Angelim (Dinizia excelsa e Andira paniculata), Angelim-Pedra (Dinizia excelsa, Hymenolobium heterocarpum, Hymenolobium petraeum e Hymenolobium pulcherrimum), Taxi (Tachigali paniculata e Tachigali myrmecophila).

Tabela 2. Estrutura horizontal das espécies inventariadas em 27 Planos de Manejo Sustentável em pequena escala no estado do Amazonas classificadas em ordem decrescente pelo Valor de Importância.

\begin{tabular}{|c|c|c|c|c|c|c|}
\hline Espécie & Familia & $\mathbf{N}$ & DR (\%) & DoR (\%) & FR (\%) & VI (\%) \\
\hline Micropholis williamii Aubrév. \& Pellegr. & Sapotaceae & 293 & 5,13 & 4,95 & 1,47 & 3,85 \\
\hline Goupia glabra Aubl. & Goupiaceae & 224 & 3,92 & 4,14 & 3,04 & 3,70 \\
\hline Couratari tauari O.Berg & Lecythidaceae & 186 & 3,25 & 5,09 & 2,25 & 3,53 \\
\hline Chrysophyllum L. & Sapotaceae & 271 & 4,74 & 4,27 & 0,90 & 3,31 \\
\hline Scleronema micranthum (Ducke) Ducke & Malvaceae & 246 & 4,30 & 3,66 & 1,92 & 3,29 \\
\hline $\begin{array}{l}\text { Licania heteromorpha var. glabra (Mart. ex } \\
\text { Hook.f.) Prance }\end{array}$ & Chrysobalanaceae & 216 & 3,78 & 3,17 & 1,92 & 2,96 \\
\hline Couepia subcordata Benth. ex Hook.f. & Chrysobalanaceae & 165 & 2,89 & 2,63 & 2,03 & 2,52 \\
\hline Tachigali paniculata Aubl. & Fabaceae & 160 & 2,80 & 2,66 & 2,03 & 2,50 \\
\hline Peltogyne densiflora Spruce ex Benth. & Fabaceae & 173 & 3,03 & 3,05 & 0,79 & 2,29 \\
\hline Dipteryx odorata (Aubl.) Willd. & Fabaceae & 127 & 2,22 & 2,47 & 2,14 & 2,28 \\
\hline Clarisia racemosa Ruiz \& Pav. & Moraceae & 126 & 2,20 & 2,02 & 2,25 & 2,16 \\
\hline Brosimum utile (Kunth) Pittier & Moraceae & 131 & 2,29 & 2,05 & 1,80 & 2,05 \\
\hline Simarouba amara Aubl. & Simaroubaceae & 123 & 2,15 & 1,90 & 2,03 & 2,03 \\
\hline Lecythis pisonis Cambess. & Lecythidaceae & 106 & 1,85 & 1,86 & 2,14 & 1,95 \\
\hline Pouteria guianensis Aubl. & Sapotaceae & 151 & 2,64 & 2,41 & 0,68 & 1,91 \\
\hline Otoba parvifolia (Markgr.) A.H.Gentry & Myristicaceae & 95 & 1,66 & 1,58 & 1,92 & 1,72 \\
\hline Aspidosperma auriculatum Markgr. & Apocynaceae & 101 & 1,77 & 1,76 & 1,24 & 1,59 \\
\hline Manilkara bidentata (A.DC.) A.Chev. & Sapotaceae & 101 & 1,77 & 1,52 & 1,24 & 1,51 \\
\hline Licaria guianensis Aubl. & Lauraceae & 71 & 1,24 & 1,16 & 2,03 & 1,48 \\
\hline Parkia nitida Miq. & Fabaceae & 70 & 1,22 & 1,31 & 1,80 & 1,44 \\
\hline Andira paniculata (Mart.) Benth. & Fabaceae & 81 & 1,42 & 2,10 & 0,79 & 1,44 \\
\hline Casearia sylvestris Sw. & Salicaceae & 85 & 1,49 & 1,34 & 1,24 & 1,36 \\
\hline Miconia poeppigii Triana & Melastomataceae & 60 & 1,05 & 0,98 & 1,92 & 1,32 \\
\hline Eschweilera pedicellata (Rich.) S.A.Mori & Lecythidaceae & 72 & 1,26 & 1,14 & 1,13 & 1,17 \\
\hline Piptadenia pteroclada Benth. & Fabaceae & 60 & 1,05 & 0,96 & 1,35 & 1,12 \\
\hline Vismia guianensis (Aubl.) Choisy & Hypericaceae & 70 & 1,22 & 1,40 & 0,68 & 1,10 \\
\hline Neea oppositifolia Ruiz \& Pav. & Nyctaginaceae & 61 & 1,07 & 0,96 & 1,24 & 1,09 \\
\hline Sextonia rubra (Mez) van der Werff & Lauraceae & 39 & 0,68 & 0,75 & 1,69 & 1,04 \\
\hline Zygia inaequalis (Willd.) Pittier & Fabaceae & 59 & 1,03 & 0,94 & 1,13 & 1,03 \\
\hline Ocotea neesiana (Miq.) Kosterm. & Lauraceae & 46 & 0,80 & 0,69 & 1,58 & 1,02 \\
\hline
\end{tabular}




\begin{tabular}{|c|c|c|c|c|c|c|}
\hline Espécie & Familia & $\mathbf{N}$ & DR (\%) & DoR (\%) & FR (\%) & VI (\%) \\
\hline Manilkara longifolia (A.DC.) Dubard & Sapotaceae & 66 & 1,15 & 1,00 & 0,90 & 1,02 \\
\hline Licania oblongifolia Standl. & Chrysobalanaceae & 53 & 0,93 & 0,91 & 1,01 & 0,95 \\
\hline Anacardium parvifolium Ducke & Anacardiaceae & 39 & 0,68 & 0,60 & 1,47 & 0,92 \\
\hline Anacardium giganteum W.Hancock ex Engl. & Anacardiaceae & 51 & 0,89 & 0,82 & 1,01 & 0,91 \\
\hline $\begin{array}{l}\text { Clinostemon mahuba (A.Samp.) Kuhlm. \& } \\
\text { A.Samp. }\end{array}$ & Lauraceae & 48 & 0,84 & 0,85 & 1,01 & 0,90 \\
\hline Terminalia amazonia (J.F.Gmel.) Exell & Combretaceae & 41 & 0,72 & 0,75 & 1,13 & 0,86 \\
\hline Theobroma L. & Malvaceae & 52 & 0,91 & 0,88 & 0,79 & 0,86 \\
\hline Amphiodon effusus Huber & Fabaceae & 52 & 0,91 & 0,86 & 0,79 & 0,85 \\
\hline Herrania mariae (Mart.) Decne. ex Goudot & Malvaceae & 55 & 0,96 & 0,80 & 0,79 & 0,85 \\
\hline Micrandra rossiana R.E.Schult. & Euphorbiaceae & 43 & 0,75 & 0,69 & 1,01 & 0,82 \\
\hline Hymenolobium pulcherrimum Ducke & Fabaceae & 44 & 0,77 & 0,64 & 1,01 & 0,81 \\
\hline Sweetia fruticosa Spreng. & Fabaceae & 48 & 0,84 & 0,90 & 0,68 & 0,81 \\
\hline Aspidosperma desmanthum Benth. ex Müll.Arg. & Apocynaceae & 28 & 0,49 & 0,49 & 1,35 & 0,78 \\
\hline Taralea oppositifolia Aubl. & Fabaceae & 44 & 0,77 & 0,76 & 0,79 & 0,77 \\
\hline Simarouba versicolor A.St.-Hil. & Simaroubaceae & 45 & 0,79 & 0,82 & 0,68 & 0,76 \\
\hline $\begin{array}{l}\text { Recordoxylon speciosum (Benoist) Gazel ex } \\
\text { Barneby }\end{array}$ & Fabaceae & 41 & 0,72 & 0,71 & 0,79 & 0,74 \\
\hline Lecythis chartacea O.Berg & Lecythidaceae & 36 & 0,63 & 0,54 & 1,01 & 0,73 \\
\hline Terminalia dichotoma G.Mey. & Combretaceae & 32 & 0,56 & 0,84 & 0,79 & 0,73 \\
\hline Caryocar glabrum (Aubl.) Pers. & Caryocaraceae & 25 & 0,44 & 0,48 & 1,24 & 0,72 \\
\hline Hymenaea oblongifolia Huber & Fabaceae & 36 & 0,63 & 0,95 & 0,56 & 0,71 \\
\hline Ocotea fragrantissima Ducke & Lauraceae & 39 & 0,68 & 0,62 & 0,79 & 0,70 \\
\hline Peltogyne paradoxa Ducke & Fabaceae & 27 & 0,47 & 0,43 & 1,01 & 0,64 \\
\hline Erisma calcaratum (Link) Warm. & Vochysiaceae & 34 & 0,59 & 0,49 & 0,79 & 0,62 \\
\hline Pouteria macrocarpa (Mart.) D.Dietr. & Sapotaceae & 50 & 0,87 & 0,75 & 0,23 & 0,62 \\
\hline Neea ovalifolia Spruce ex J.A.Schmidt & Nyctaginaceae & 33 & 0,58 & 0,53 & 0,68 & 0,60 \\
\hline Eschweilera Mart. ex DC. & Lecythidaceae & 33 & 0,58 & 0,49 & 0,68 & 0,58 \\
\hline Couepia bracteosa Benth. & Chrysobalanaceae & 26 & 0,45 & 0,52 & 0,68 & 0,55 \\
\hline Talisia acutifolia Radlk. & Sapindaceae & 18 & 0,31 & 0,62 & 0,68 & 0,54 \\
\hline Lecythis Loefl. & Lecythidaceae & 23 & 0,40 & 0,39 & 0,79 & 0,53 \\
\hline Couratari Aubl. & Lecythidaceae & 26 & 0,45 & 0,38 & 0,68 & 0,50 \\
\hline Couepia robusta Huber & Chrysobalanaceae & 21 & 0,37 & 0,63 & 0,45 & 0,48 \\
\hline Brosimum parinarioides Ducke & Moraceae & 19 & 0,33 & 0,40 & 0,68 & 0,47 \\
\hline Piptadenia gonoacantha (Mart.) J.F.Macbr. & Fabaceae & 17 & 0,30 & 0,58 & 0,45 & 0,44 \\
\hline Mezilaurus itauba (Meisn.) Taub. ex Mez & Lauraceae & 15 & 0,26 & 0,25 & 0,79 & 0,43 \\
\hline Lecythis usitata Miers & Lecythidaceae & 18 & 0,31 & 0,37 & 0,56 & 0,42 \\
\hline Vatairea guianensis Aubl. & Fabaceae & 18 & 0,31 & 0,23 & 0,68 & 0,41 \\
\hline Theobroma martiana D.Dietr. & Malvaceae & 25 & 0,44 & 0,44 & 0,34 & 0,41 \\
\hline Licaria chrysophylla (Meisn.) Kosterm. & Lauraceae & 16 & 0,28 & 0,24 & 0,68 & 0,40 \\
\hline Caryocar L. & Caryocaraceae & 19 & 0,33 & 0,40 & 0,45 & 0,40 \\
\hline $\begin{array}{l}\text { Staminodianthus racemosus (Hoehne) } \\
\text { D.B.O.S.Cardoso \& H.C.Lima }\end{array}$ & Fabaceae & 14 & 0,24 & 0,24 & 0,68 & 0,39 \\
\hline Manilkara elata (Allemão ex Miq.) Monach. & Sapotaceae & 10 & 0,17 & 0,20 & 0,79 & 0,39 \\
\hline Eschweilera coriacea (DC.) S.A.Mori & Lecythidaceae & 21 & 0,37 & 0,31 & 0,45 & 0,38 \\
\hline Lecythis zabucajo Aubl. & Lecythidaceae & 14 & 0,24 & 0,31 & 0,56 & 0,37 \\
\hline Caryocar microcarpum Ducke & Caryocaraceae & 17 & 0,30 & 0,25 & 0,56 & 0,37 \\
\hline
\end{tabular}




\begin{tabular}{|c|c|c|c|c|c|c|}
\hline Espécie & Familia & $\mathbf{N}$ & DR (\%) & DoR (\%) & FR (\%) & VI (\%) \\
\hline Endopleura uchi (Huber) Cuatrec. & Humiriaceae & 12 & 0,21 & 0,31 & 0,56 & 0,36 \\
\hline Inga paraensis Ducke & Fabaceae & 22 & 0,38 & 0,34 & 0,34 & 0,35 \\
\hline Caryocar villosum (Aubl.) Pers. & Caryocaraceae & 9 & 0,16 & 0,33 & 0,56 & 0,35 \\
\hline Pleurothyrium cuneifolium Nees & Lauraceae & 15 & 0,26 & 0,31 & 0,45 & 0,34 \\
\hline Acioa edulis Prance & Chrysobalanaceae & 15 & 0,26 & 0,49 & 0,23 & 0,33 \\
\hline Licania hypoleuca Benth. var. hypoleuca & Chrysobalanaceae & 12 & 0,21 & 0,20 & 0,56 & 0,33 \\
\hline Hymenolobium petraeum Ducke & Fabaceae & 8 & 0,14 & 0,13 & 0,68 & 0,31 \\
\hline Calycophyllum spruceanum (Benth.) K.Schum. & Rubiaceae & 15 & 0,26 & 0,22 & 0,45 & 0,31 \\
\hline Dinizia excelsa Ducke & Fabaceae & 22 & 0,38 & 0,30 & 0,23 & 0,30 \\
\hline Aiouea Aubl. & Lauraceae & 13 & 0,23 & 0,22 & 0,45 & 0,30 \\
\hline Bowdichia virgilioides Kunth & Fabaceae & 11 & 0,19 & 0,20 & 0,45 & 0,28 \\
\hline Hymenaea parvifolia Huber & Fabaceae & 7 & 0,12 & 0,15 & 0,56 & 0,28 \\
\hline Tachigali glauca Tul. & Fabaceae & 16 & 0,28 & 0,32 & 0,23 & 0,28 \\
\hline Brosimum rubescens Taub. & Moraceae & 11 & 0,19 & 0,18 & 0,45 & 0,27 \\
\hline Iryanthera paraensis Huber & Myristicaceae & 10 & 0,17 & 0,28 & 0,34 & 0,26 \\
\hline Erythrina fusca Lour. & Fabaceae & 6 & 0,10 & 0,12 & 0,56 & 0,26 \\
\hline $\begin{array}{l}\text { Osteophloeum platyspermum (Spruce ex A.DC.) } \\
\text { Warb. }\end{array}$ & Myristicaceae & 20 & 0,35 & 0,27 & 0,11 & 0,24 \\
\hline Vouacapoua pallidior Ducke & Fabaceae & 7 & 0,12 & 0,23 & 0,34 & 0,23 \\
\hline Scleronema praecox (Ducke) Ducke & Malvaceae & 20 & 0,35 & 0,21 & 0,11 & 0,22 \\
\hline Cariniana micrantha Ducke & Lecythidaceae & 8 & 0,14 & 0,42 & 0,11 & 0,22 \\
\hline Platonia insignis Mart. & Clusiaceae & 4 & 0,07 & 0,15 & 0,45 & 0,22 \\
\hline Ocotea longifolia Kunth & Lauraceae & 8 & 0,14 & 0,16 & 0,34 & 0,21 \\
\hline Nectandra discolor (Kunth) Nees & Lauraceae & 16 & 0,28 & 0,23 & 0,11 & 0,21 \\
\hline Aniba terminalis Ducke & Lauraceae & 9 & 0,16 & 0,12 & 0,34 & 0,21 \\
\hline $\begin{array}{l}\text { Handroanthus capitatus (Bureau \& K.Schum.) } \\
\text { Mattos }\end{array}$ & Bignoniaceae & 5 & 0,09 & 0,06 & 0,45 & 0,20 \\
\hline Não identificada & $\mathrm{NI}$ & 8 & 0,14 & 0,10 & 0,34 & 0,19 \\
\hline Iryanthera polyneura Ducke & Myristicaceae & 9 & 0,16 & 0,29 & 0,11 & 0,19 \\
\hline Vochysia maxima Ducke & Vochysiaceae & 2 & 0,03 & 0,38 & 0,11 & 0,18 \\
\hline Duckesia verrucosa (Ducke) Cuatrec. & Humiriaceae & 9 & 0,16 & 0,12 & 0,23 & 0,17 \\
\hline Eschweilera grandiflora (Aubl.) Sandwith & Lecythidaceae & 14 & 0,24 & 0,15 & 0,11 & 0,17 \\
\hline Hymenolobium heterocarpum Ducke & Fabaceae & 4 & 0,07 & 0,09 & 0,34 & 0,17 \\
\hline Virola guggenheimii W.A.Rodrigues & Myristicaceae & 5 & 0,09 & 0,06 & 0,34 & 0,16 \\
\hline Chrysophyllum prieurii A.DC. & Sapotaceae & 10 & 0,17 & 0,16 & 0,11 & 0,15 \\
\hline Hymenaea courbaril L. & Fabaceae & 7 & 0,12 & 0,09 & 0,23 & 0,15 \\
\hline Theobroma speciosa Willd. ex Mart. & Malvaceae & 10 & 0,17 & 0,14 & 0,11 & 0,14 \\
\hline Ocotea caudata Mez. & Lauraceae & 6 & 0,10 & 0,08 & 0,23 & 0,14 \\
\hline Brosimum angustifolium Ducke & Moraceae & 6 & 0,10 & 0,08 & 0,23 & 0,14 \\
\hline Tabebuia barbata (E.Mey.) Sandwith & Bignoniaceae & 4 & 0,07 & 0,11 & 0,23 & 0,13 \\
\hline Bowdichia nitida Spruce ex Benth. & Fabaceae & 7 & 0,12 & 0,14 & 0,11 & 0,13 \\
\hline Iryanthera olacoides A.C.Sm. & Myristicaceae & 11 & 0,19 & 0,07 & 0,11 & 0,12 \\
\hline Swartzia polyphylla DC. & Fabaceae & 7 & 0,12 & 0,11 & 0,11 & 0,11 \\
\hline Manilkara salzmannii (A.DC.) H.J.Lam & Sapotaceae & 3 & 0,05 & 0,05 & 0,23 & 0,11 \\
\hline Cassia fastuosa Willd. ex Benth. & Fabaceae & 5 & 0,09 & 0,13 & 0,11 & 0,11 \\
\hline Dalbergia spruceana Benth. & Fabaceae & 2 & 0,03 & 0,03 & 0,23 & 0,10 \\
\hline
\end{tabular}




\begin{tabular}{|c|c|c|c|c|c|c|}
\hline Espécie & Familia & $\mathbf{N}$ & DR (\%) & DoR (\%) & FR (\%) & VI (\%) \\
\hline Leptolobium nitens Vogel & Fabaceae & 2 & 0,03 & 0,03 & 0,23 & 0,10 \\
\hline Bagassa guianensis Aubl. & Moraceae & 5 & 0,09 & 0,08 & 0,11 & 0,09 \\
\hline Brosimum potabile Ducke & Moraceae & 4 & 0,07 & 0,10 & 0,11 & 0,09 \\
\hline Licania heteromorpha Benth. & Chrysobalanaceae & 6 & 0,10 & 0,05 & 0,11 & 0,09 \\
\hline Bellucia dichotoma Cogn. & Melastomataceae & 4 & 0,07 & 0,08 & 0,11 & 0,09 \\
\hline Sapium ciliatum Hemsl. & Euphorbiaceae & 4 & 0,07 & 0,06 & 0,11 & 0,08 \\
\hline Lecythis idatimon Aubl. & Lecythidaceae & 4 & 0,07 & 0,06 & 0,11 & 0,08 \\
\hline Peltogyne catingae Ducke & Fabaceae & 4 & 0,07 & 0,05 & 0,11 & 0,08 \\
\hline Moronobea pulchra Ducke & Clusiaceae & 4 & 0,07 & 0,04 & 0,11 & 0,07 \\
\hline Ocotea cymbarum Kunth & Lauraceae & 3 & 0,05 & 0,06 & 0,11 & 0,07 \\
\hline Astronium lecointei Ducke & Anacardiaceae & 2 & 0,03 & 0,07 & 0,11 & 0,07 \\
\hline Peltogyne venosa Benth & Fabaceae & 3 & 0,05 & 0,05 & 0,11 & 0,07 \\
\hline Cedrela odorata L. & Meliaceae & 2 & 0,03 & 0,05 & 0,11 & 0,07 \\
\hline Persea caerulea (Ruiz \& Pav.) Mez & Lauraceae & 3 & 0,05 & 0,03 & 0,11 & 0,06 \\
\hline Calophyllum brasiliense Cambess. & Calophyllaceae & 2 & 0,03 & 0,04 & 0,11 & 0,06 \\
\hline Byrsonima chrysophylla Kunth & Malpighiaceae & 2 & 0,03 & 0,04 & 0,11 & 0,06 \\
\hline Buchenavia viridiflora Ducke & Combretaceae & 2 & 0,03 & 0,04 & 0,11 & 0,06 \\
\hline Tabebuia Gomes ex DC. & Bignoniaceae & 2 & 0,03 & 0,04 & 0,11 & 0,06 \\
\hline Guarea kunthiana A.Juss. & Meliaceae & 2 & 0,03 & 0,04 & 0,11 & 0,06 \\
\hline Acrodiclidium appelli Mez. & Lauraceae & 2 & 0,03 & 0,03 & 0,11 & 0,06 \\
\hline Sextonia rubra (Mez) van der Werff & Lauraceae & 2 & 0,03 & 0,03 & 0,11 & 0,06 \\
\hline Cariniana Casar. & Lecythidaceae & 2 & 0,03 & 0,03 & 0,11 & 0,06 \\
\hline Couma guianensis Aubl. & Apocynaceae & 2 & 0,03 & 0,02 & 0,11 & 0,06 \\
\hline Brosimum rubescens Taub. & Moraceae & 1 & 0,02 & 0,03 & 0,11 & 0,05 \\
\hline Cordia incognita Gottschling \& J.S.Mill. & Boraginaceae & 1 & 0,02 & 0,02 & 0,11 & 0,05 \\
\hline Pseudobombax munguba (Mart.) Dugand & Malvaceae & 1 & 0,02 & 0,02 & 0,11 & 0,05 \\
\hline Maquira sclerophylla (Ducke) C.C.Berg & Moraceae & 1 & 0,02 & 0,02 & 0,11 & 0,05 \\
\hline Rinorea guianensis Aubl. & Violaceae & 1 & 0,02 & 0,02 & 0,11 & 0,05 \\
\hline Anacardium spruceanum Benth. ex Engl. & Anacardiaceae & 1 & 0,02 & 0,02 & 0,11 & 0,05 \\
\hline Cariniana domestica (Mart.) & Lecythidaceae & 1 & 0,02 & 0,02 & 0,11 & 0,05 \\
\hline Euxylophora paraensis Huber & Rutaceae & 1 & 0,02 & 0,02 & 0,11 & 0,05 \\
\hline Swartzia leptopetala Benth. & Fabaceae & 1 & 0,02 & 0,02 & 0,11 & 0,05 \\
\hline Erisma Rudge & Vochysiaceae & 1 & 0,02 & 0,01 & 0,11 & 0,05 \\
\hline Xylopia L. & Annonaceae & 1 & 0,02 & 0,01 & 0,11 & 0,05 \\
\hline Senna multijuga (Rich.) H.S.Irwin \& Barneby & Fabaceae & 1 & 0,02 & 0,01 & 0,11 & 0,05 \\
\hline Piranhea trifoliata Baill. & Picrodendraceae & 1 & 0,02 & 0,01 & 0,11 & 0,05 \\
\hline Aniba guianensis Aubl. & Lauraceae & 1 & 0,02 & 0,01 & 0,11 & 0,05 \\
\hline Protium araguense Cuatrec. & Burseraceae & 1 & 0,02 & 0,01 & 0,11 & 0,05 \\
\hline $\begin{array}{l}\text { Anacardium excelsum (Bertero \& Balb. ex } \\
\text { Kunth) Skeels }\end{array}$ & Anacardiaceae & 1 & 0,02 & 0,01 & 0,11 & 0,05 \\
\hline Schizolobium amazonicum Ducke & Fabaceae & 1 & 0,02 & 0,01 & 0,11 & 0,05 \\
\hline Total & & 5716 & 100,00 & 100,00 & 100,00 & 100,00 \\
\hline
\end{tabular}

O índice de diversidade de Shannon foi de 4,28, indicando alta diversidade de acordo com Knight (1975), sendo que esse valor é semelhante e até superior a outros calculados em florestas na Amazônia (SILVA et al., 2011; CONDÉ \& TONINI, 2013; CARIM et al., 2015; BRANDÃO et al., 2020; VINHOTE et al., 2020). O índice de Equabilidade 
de Pielou foi de 0,85. Esse índice indica dominância máxima ou mínima de uma espécie, com valores variando de 0 a 1 , sendo que 0 indica que todos os indivíduos pertencem à mesma espécie (diversidade mínima), e 1 que cada indivíduo pertence a espécies diferentes (diversidade máxima). Dessa forma, não há a dominância de uma espécie nos planos de manejo considerados.

A estrutura diamétrica dos dados não apresentou o padrão de "J-invertido", como é esperado para levantamentos florísticos na região amazônica (Figura 1). Entretanto, esse comportamento é explicado pela não padronização do diâmetro mínimo de inclusão nos inventários florestais realizados. Em apenas três inventários os menores diâmetros são entre 20 e $30 \mathrm{~cm}$. Por outro lado, em todos os inventários há indivíduos com DAP menor que $50 \mathrm{~cm}$, entretanto, não foram medidas todas as árvores, uma vez que o diâmetro mínimo de corte permitido em legislação é de $50 \mathrm{~cm}$, dessa forma, optou-se pela concentração da coleta de dados das árvores com diâmetros a partir desse valor, a fim de otimizar o tempo e, consequentemente, os recursos disponíveis. Destaca-se o bom número de indivíduos verificados com maior de 100 $\mathrm{cm}$ de diâmetro, o que pode proporcionar boas condições econômicas para o manejo florestal.

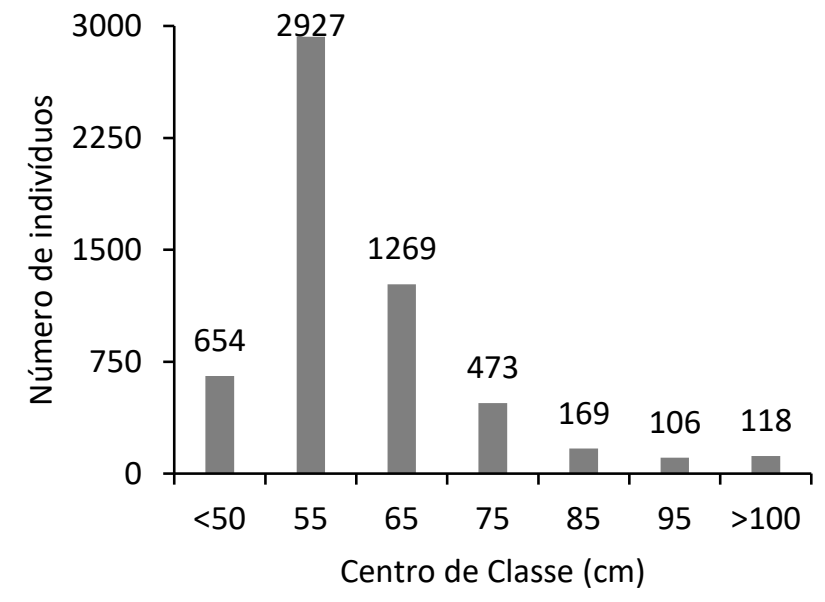

Figura 1. Número de indivíduos por classe de diâmetro em planos de manejo florestal sustentáveis em pequena escala no estado do Amazonas.

\section{CONCLUSÕES}

As espécies de maior interesse para pequenos produtores no Amazonas para exploração madeireira são Abiurana, Cupiúba, Tauari, Cedrinho, Macucu, Marirana, Taxi, Roxinho e Cumaru.

A diversidade de espécies em planos de manejo em pequena escala condiz com o esperado para a região amazônica, sendo mais elevado que outros estudos na região.

A padronização das metodologias de inventário florestal realizados para a elaboração de PMFSPE podem proporcionar um conhecimento mais detalhado sobre a composição de espécies na região.

\section{AGRADECIMENTOS}

Agradecemos ao Instituto de Desenvolvimento Agropecuário e Florestal Sustentável do Estado do Amazonas pela disponibilização dos dados necessários para a realização desse estudo.

\section{REFERÊNCIAS}

AMAZONAS. Conselho Estadual de Meio Ambiente do Estado do Amazonas. Resolução no 007, de 21 de junho de 2011. Diário Oficia do Estado, Manaus, 2011.

ANDRADE, D.F. et al. Inventário Florestal de Grandes Áreas na Floresta Nacional do Tapajós, Pará, Amazônia, Brasil. Biota Amazônia, v.5, n.1, p.109-115, 2015.

BRASIL. Lei no 4.771, de 15 de setembro de 1965. Diário Oficial da União. Brasília, 1965.

BRASIL. Lei no 12.651, de 25 de maio de 2012. Diário Oficial da União. Brasília, 2012.

BRANDÃO, P.C. et al. Caracterização estrutural e potencial florestal para o manejo comunitário da Floresta Nacional do Purus, Amazônia Ocidental. Ciência Florestal, v.30, n.4, p.944$957,2020$.

CARIM, M.J.V. et al. Composition, structure and floristic diversity in dense rain forest in the Eastern Amazon, Amapá, Brazil. Acta Scientiarum, v.37, n.4, p.419-26, 2015.

CONDÉ, T.M.; TONINI, H. Fitossociologia de uma Floresta Ombrófila Densa na Amazônia Setentrional, Roraima, Brasil. Acta Amazonica, v.43, n.3, p.247-260, 2013.

FREITAS, F.C. Fitossociologia e distribuição geográfica de espécies arbóreas licenciadas em planos de manejo florestal sustentável em pequena escala assistidos pelo instituto de desenvolvimento agropecuário e florestal sustentável do estado do Amazonas. 2014. 102p. (Monografia de Graduação).

IBGE. Manual técnico da vegetação brasileira. Rio de Janeiro, 2012.

KLIBER, J.F. Manual técnico sobre planos de manejo florestal sustentável em pequena escala - PMFSPE. Manaus: Floresta Viva, 2008.

JARDIM, F.C.S.; QUADROS, L.C.L. Estrutura de uma floresta tropical dez anos após exploração de madeira em Moju, Pará. Ceres, v.63, n.4, p.427-435, 2016.

KNIGHT, D.H. A phytosociological analysis of species-rich tropical 
forest on Barro Colorado Island, Panama. Ecological Monographs, v.45, p.259-284, 1975.

LIMA, B.D.A. et al. Estrutura e dinâmica florestal sob efeito do manejo madeireiro na FLONA Tapajós. Advances in Forestry Science, v.5, n.4, p.437-443, 2018.

LIMA, R.B.A. et al. Fitossociologia de um trecho de floresta ombrófila densa na Reserva de Desenvolvimento Sustentável Uacari, Carauari, Amazonas. Scientia Plena, v.9, n.1, p.1-12, 2012.

MUELLER-DOMBOIS, D.; ELLENBERG, H. Aims and methods of vegetation ecology. New York: John Wiley \& Sons, 1974.

OLIVEIRA, A.N.; AMARAL, I.L. Florística e fitossociologia de uma floresta de vertente na Amazônia Central, Amazonas, Brasil. Acta Amazônica, v 34, n.1, p.21-34, 2004.

PIELOU, E.C. The measurement of diversity in different types of biological collections. Journal of Theoretical Biology, v.13, p.131144, 1966.

SHANNON, C.E. A mathematical theory of communication. Bell System Technical Journal, v.27, p.379-423, 1948.

SILVA, K.E. et al. Floristic composition and similarity of 15 hectares in Central Amazon, Brazil. Revista de Biología Tropical, v.59, n.4, p.1927-1938, 2011.

STEEGE, H. et al. Hyperdominance in the Amazonian Tree Flora. Science, v.342, p.6159, 2013.

VIEIRA, D.S. et al. Comparação estrutural entre floresta manejada e não manejada na comunidade santo antônio, estado do pará. Ciencia Florestal, v.24, n.4, p.1061-1068, 2014.

VINHOTE, E.G. et al. Diversity and similarity of species of natural regeneration after logging in commercially managed forest in Central Amazon. Ciencia Florestal, v.30, n.4, p.1116-1129, 2020.

Recebido em 04-08-2021 Aceito em 25-10-2021 